\title{
The large-scale radio spectral index distribution of Centaurus A
}

\author{
J.A. Combi ${ }^{\star}$ and G.E. Romero ${ }^{\star}$ \\ Instituto Argentino de Radioastronomía, C.C. 5, (1894) Villa Elisa, Prov. de Bs. As., Argentina \\ Received June 8, 1995; accepted April 13, 1996
}

\begin{abstract}
A spectral index map of Centaurus A (Cen A) has been computed using $408 \mathrm{MHz}$ observations from the whole-sky survey by Haslam et al. (1981) and new 1435 $\mathrm{MHz}$ observations. The large-scale structure of the radio source is discussed in the light of these new data and a previous polarization survey. The observational evidence seems to support the expanding jet model for this source.
\end{abstract}

Key words: galaxies: individual: NGC $5128=$ Cen A galaxies: active - radio continuum: galaxies

\section{Introduction}

Cen $\mathrm{A}$ is the nearest active galaxy. At radio frequencies it appears as an extended and very strong source, with a VLBI-core, a one-sided jet, Double Inner Lobes, a Northern Middle Lobe and two Giant Outer Lobes (see Ebneter \& Balick 1983 for details). The large-scale radio structure has been studied by several authors: Cooper et al. (1965), Haynes et al. (1983), and recently Junkes et al. (1993). The latter have presented results of a $\lambda 6.3 \mathrm{~cm}$ continuum and polarization survey of the whole source obtained with the Parkes radiotelescope. Their work will be completed by similar radio observations at $\lambda 3 \mathrm{~cm}$ whose analysis will provide information about the spectral index distribution, rotation measures and depolarization. Here we present some complementary data to these studies. By means of the $408 \mathrm{MHz}$ continuum observations carried out by Haslam et al. (1981) and new $1435 \mathrm{MHz}$ observations we have computed a spectral index map of the extended source. This map, together with the polarization maps presented by Junkes and co-workers, can provide valuable information about the physical processes occurring in the large-scale structure of Cen A. Previous computations of the spectral indices of the extended radio emission in this source were restricted to mean values (Cooper et al. 1965).

Send offprint requests to: J.A. Combi

* Fellow of CONICET

\section{Observations and data reduction}

The observations were performed with one of the $30-\mathrm{m}$ telescopes of the Instituto Argentino de Radioastronomía (IAR) in May 1992. The two-channel receiver was operated at a central frequency of $1435 \mathrm{MHz}$ with a bandwidth of $20 \mathrm{MHz}$. The temperature of the system was $90 \mathrm{~K}$. The HPBW of the antenna is 30 arcmin at this frequency, and the $T_{\mathrm{B}} / S$ ratio of the main beam brightness temperature to the flux density was $0.16 \mathrm{~K} \mathrm{Jy}^{-1}$ during the observations.

The observing technique consisted in fast $\left(10^{\circ} / \mathrm{min}\right)$ scans in declination, regularly spaced in right ascension. Two "up" and two "down" scans were performed at each selected value of right ascension. The standard sources Hydra A and PKS 1308-22 were observed for calibration purposes. The data reduction was carried out following the techniques described by Combi et al. (1995) and Combi \& Romero (1995), which are based on those developed by Haslam et al. (1974). The data from the survey by Haslam et al. (1981) were processed in the same way as the new $1435 \mathrm{MHz}$ data. In order to eliminate local contributions a smooth background was subtracted out by applying the 'background filtering' method developed by Sofue \& Reich (1979). The diffuse emission subtracted consists of two components: a galactic disk contribution and a component resulting from the merging of unresolved smallscale background sources, mainly extragalactic ones. This subtraction technique, however, can not suppress the local contribution of the spur-like feature which appears to point out towards the southern part of the galaxy (Haslam et al. 1981). Owing to the strength of Cen A at both observing frequencies we estimate that the total contribution of the spur is negligible. A complete study of this galactic structure is in progress in order to determine its nature and spectral characteristics (Combi \& Romero, in preparation).

The resulting radio continuum maps (see Fig. 1, where the brightest part of the spur is visible on the left) were used for computing the spectral index distribution in the source, after convolution and re-tabulation of the 

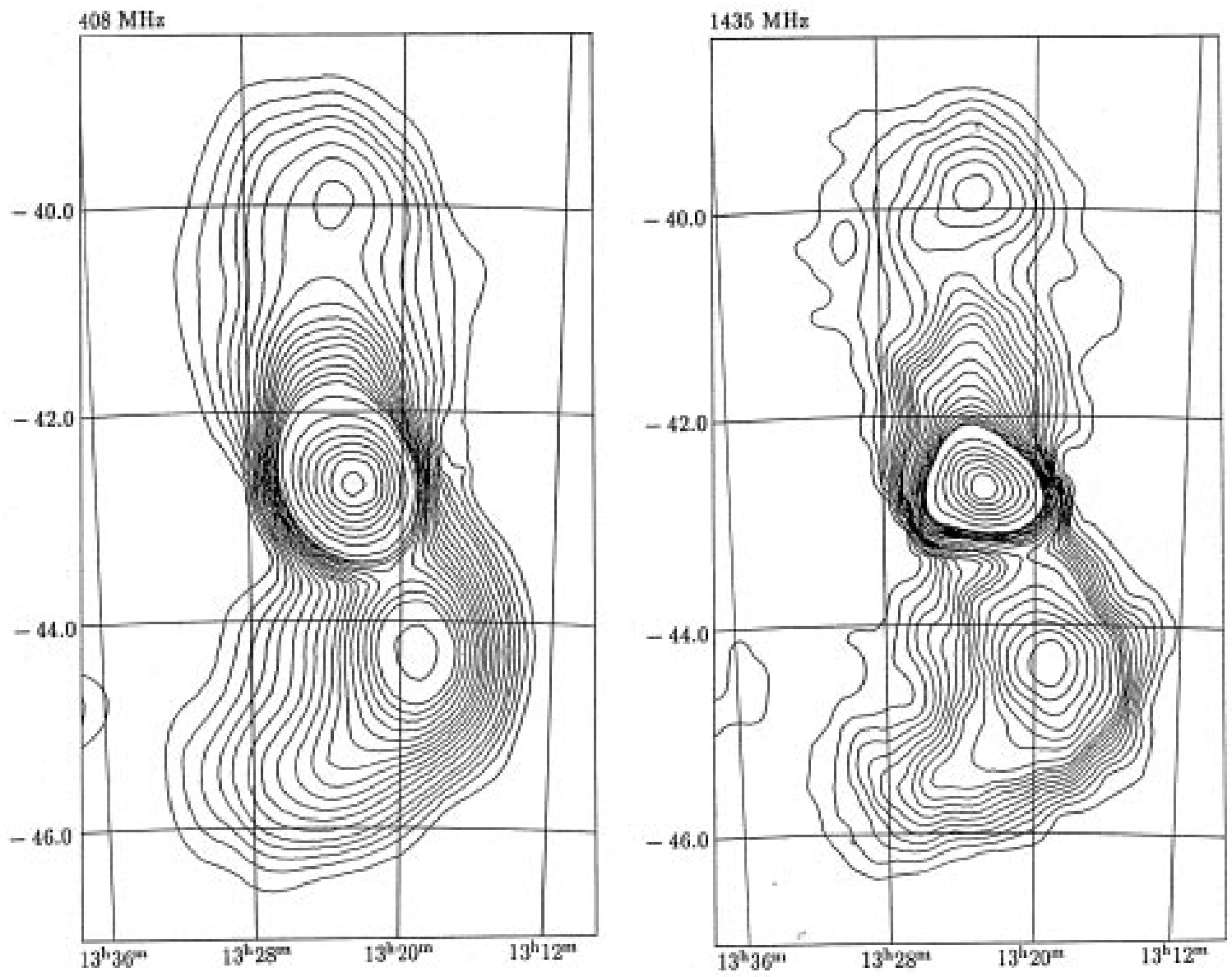

Fig. 1. a) Radio continuum emission at $408 \mathrm{MHz}$ from Cen $\mathrm{A}$ (with the original beam) after subtraction of the smooth 'backgound' emission. Contour lines are shown at $1,5, \ldots ., 20 ; 25,31, \ldots ., 67 ; 75,83, \ldots ., 107 ; 120,170, \ldots . ., 500 \mathrm{~K}$, in brightness temperature. b) Radio continuum emission at $1435 \mathrm{MHz}$ from Cen A (with the original beam) after subtraction of the smooth 'background' emission. Contour lines are shown at $0.1,0.2, \ldots . ., 1 ; 1.35,1.65, \ldots ., 4 ; 6,8, \ldots . ., 18 ; 21,24, \ldots ., 32 \mathrm{~K}$. Coordinates are referred to 1950.0

$1435 \mathrm{MHz}$ map to the same beam and tabular interval of the $408 \mathrm{MHz}$ map. Two extragalactic compact radio sources, 1332-33 and 1334-127 were used for checking the positional accuracy of the maps by comparing the measured positions with the actual positions taken from the 1 Jy Catalogue by Kühr et al. (1981). The second source, the flat-spectrum QSO 1334-127, was also used for matching the different beam shapes.

The spectral index $\alpha$ (we consider $S \propto \nu^{\alpha}$ ) between $408 \mathrm{MHz}$ and $1435 \mathrm{MHz}$ was computed as:

$\alpha=2-\log \left(\frac{T_{408}}{T_{1435}}\right) / \log \left(\frac{1435}{408}\right)$

where $T_{\nu}$ is the brightness temperature at the frequency $\nu$ obtained after the removal of the diffuse galactic contribution (see Combi et al. 1995 for details about the procedure). The reliability of the spectral index computation rests on the determination of the absolute zero level of each set of data under consideration. Systematic errors can be introduced by variations of the zero level error to intensity ratio with frequency. In order to get an accurate estimate of these errors we have followed the procedure described by Reich \& Reich (1988). The errors were determined as:

$\triangle \alpha \approx\left[\left(\triangle T_{\mathrm{b}} / T_{\mathrm{b}}\right)_{1435}^{2}+\left(\triangle T_{\mathrm{b}} / T_{\mathrm{b}}\right)_{408}^{2}\right] / \ln (1435 / 408)$,

where the contributing errors to $\triangle T_{\mathrm{b}}$ are the result of scale errors, local scanning errors, local noise, and the error of the absolute zero level. Values for the $408 \mathrm{MHz}$ data are quoted by Reich \& Reich (1988) in their Table VIII. Errors for our own data are of the same order as those described in that Table for the $1420 \mathrm{MHz}$ observations, except for $\triangle T_{\text {rms }}$, which is in our case $\sim 0.02 \mathrm{~K}$. We estimate the maximum error in our final map as $|\triangle \alpha|_{\max } \approx 0.16$. The mean error in the map is $|\triangle \alpha|_{\text {mean }} \approx 0.08$. The errors in the Southern Lobe could be slightly underestimated due to the mentioned weak contribution of the local spur structure. 


\section{Results}

Figure 2 shows the spectral index distribution between $408 \mathrm{MHz}$ and $1435 \mathrm{MHz}$ for Cen A. Three levels can be appreciated in the map. About $58 \%$ of the emission is in the range $-0.7 \leq \alpha \leq-0.5$. The rest of the emission has steeper indices: $28 \%$ is in the $-0.9 \leq \alpha \leq-0.7$ range, and the remaining $14 \%$ is in the $-1.3 \leq \alpha \leq-0.9$ range. These values are in agreement with the average nonthermal index reported by Cooper et al. (1965).

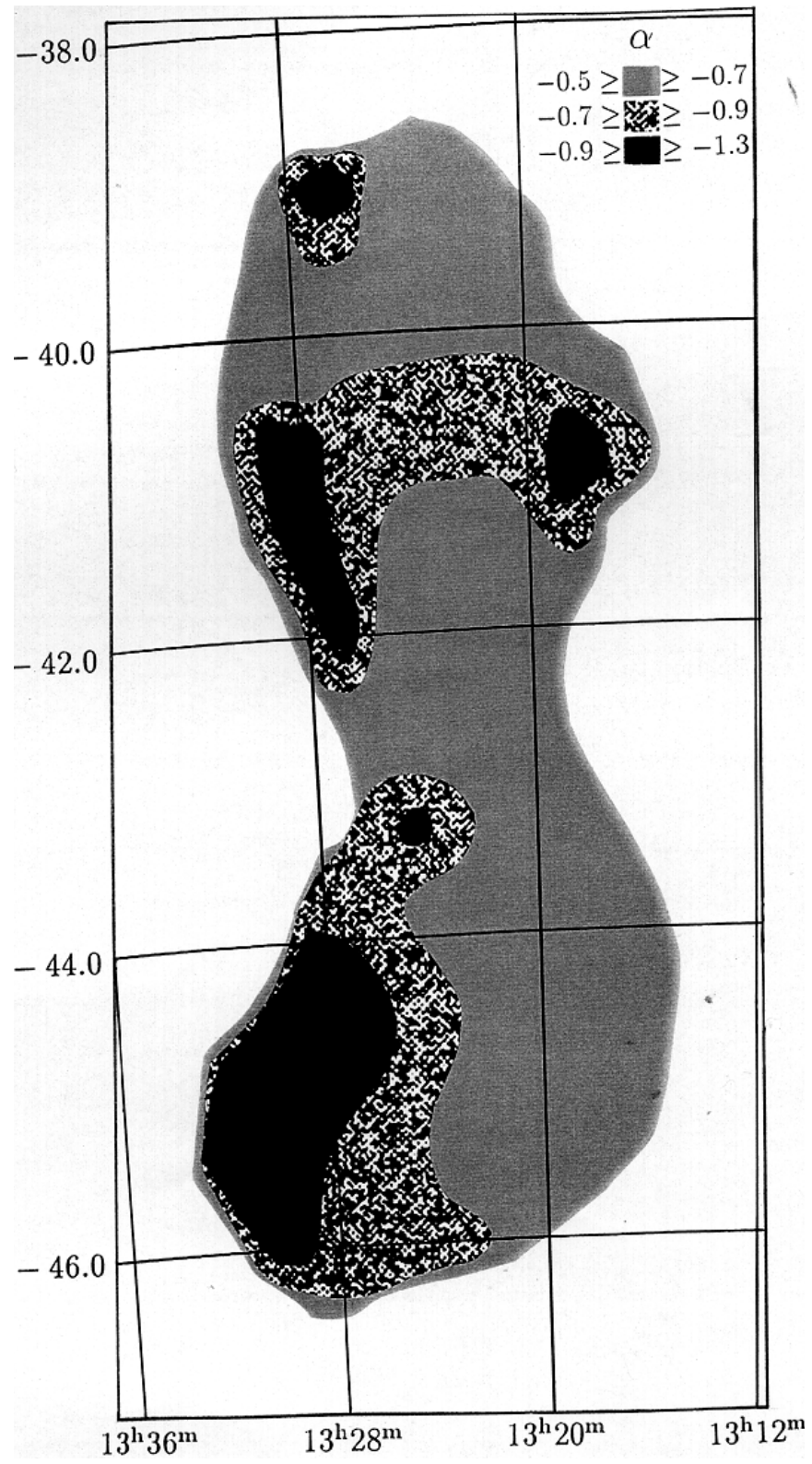

Fig. 2. Spectral index map for Cen A computed between $408 \mathrm{MHz}$ and $1435 \mathrm{MHz}$. Coordinates are referred to 1950.0

The index distribution is different in both Giant Lobes. The Northern Giant Lobe presents three regions with steep indices located towards its outer borders. The Northern Middle Lobe has similar values to those found in the Northern Loop (see Junkes et al. 1993 for details about this latter feature). The Double Inner Lobes share these values (in the $-0.7 \leq \alpha \leq-0.5$ range). In the Southern Giant Lobe there is a steepening of the indices towards increasing values of right ascension. The steepest values $(\alpha \sim-1.3)$ are in the southeast part of the source.

When our map is compared with the polarization map obtained by Junkes et al. (1993) it is easy to see that the regions with indices in the $-1.3 \leq \alpha \leq-0.9$ range are unpolarized. The higher polarized intensities comes from regions with $-0.7 \leq \alpha \leq-0.5$, in particular from the Northern Middle Lobe and the Double Inner Lobes.

\section{Comments}

The spectral indices in Cen A are typical for nonthermal emission produced by the synchrotron mechanism. A relativistic electron population with a power law energy spectrum $N(E)=N_{0} E^{-p}$ will produce a flux density $S(\nu) \propto N_{0} B_{0}^{(1+p) / 2} \nu^{(1-p) / 2}$, for an optically thin source with uniform magnetic field $B_{0}$. In this case, the degree of linear polarization will be approximately (e.g. Pacholczyk 1970):

$m_{0}(p)=\frac{3 p+3}{3 p+7}$

where $p$ is related with the observed spectral index by $p=$ $1-2 \alpha$. In order to account for the polarization reported by Junkes et al. (1993) a random magnetic field $B_{\mathrm{r}}$ must be present in the source. Then, the degree of polarization becomes:

$m(p)=m_{0}(p) \frac{B_{0}^{2}}{B_{0}^{2}+B_{\mathrm{r}}^{2}}$

Using the spectral indices presented in this paper we find $B_{\mathrm{r}} \sim 0.6 B_{0}$ in the Double Inner Lobes and in the Northern Middle Lobe. In the steepest regions of the Giant Lobes the random field is $B_{\mathrm{r}} \sim 0.7 B_{0}$.

Several models have been invoked to explain the largescale radio appearence of Cen A (e.g. Blandford \& Rees 1974; Kellerman \& Pauliny-Toth 1981, van Albada et al. 1982; Quinn 1982). The recent work by Junkes et al. (1993), which established that the Nothern and Southern Giant Lobes are linked by a bridge of continuum emission, together with the nonthermal spectral index distribution displayed in Fig. 2 seem to support the expanding jet model with induced shocks as a method for powering the extended radio source. This model is also supported by evidence of ongoing activity in the nucleus (Kellermann 1974; Morganti et al. 1992; Botti et al. 1993), and by X-ray, optical and radio observations of the inner jet of the source (Schreier et al. 1979; Feigelson et al. 1981; Burns et al. 1983; Haynes et al. 1983; Meier et al. 1989). 
Summarizing, the large-scale radio emission of Cen A seems to agree with the picture of an active nucleus with a jet forming a relatively small angle with the line of sight. This jet, which can be directly observed at VLA scales, would be responsible for the formation of the Northern Inner and Middle Lobes when it interacts with the interstellar and intergalactic medium, respectively. The Northern Giant Lobe would be the cocoon associated to the Middle Lobe. Finally, the southern Inner and Giant Lobes would be produced by a counter jet and, consequently, they would be situated farther from the observer than the northern lobes. This fact seems to be reflected by the lower degree of polarization observed in the southern components.

Acknowledgements. We thank P. Benaglia for his critical reading of the manuscript. This work was partially supported by CONICET and the Universidad Nacional de La Plata.

\section{References}

Blandford R.D., Rees M.J., 1974, MNRAS 169, 395

Botti L.C.L., Abraham Z., 1993, MNRAS 264, 807

Burns J.O., Fiegelson E.D., Schreier E.J., 1983, ApJ 273, 128

Combi J.A., Testori J.C., Romero G.E., Colomb F.R., 1995, A\&A 296, 514
Combi J.A., Romero G.E., 1995, A\&A 303, 872

Cooper B.F.C., Price R.M., Cole D.J., 1965, Aust. J. Phys. 18, 589

Ebneter K., Balick B., 1983, PASP 95, 675

Feigelson E.D., 1981, ApJ 251, 31

Haslam C.G.T., Wilson W.E., Graham D.A., Hunt G.C., 1974, A\&AS 13,359

Haslam C.G.T., Klein U., Salter C.J., et al., 1981, A\&A 100, 209

Haynes R.F., Cannon R.D., Ekers R.D., 1983, Proc. ASA 5, 241

Junkes N., Haynes R.F., Harnett J.I., Jauncey D.L., 1993, A\&A 269, 29

Kellerman K.I., 1974, ApJ 194, L135

Kellerman K.I., Pauliny-Toth I.I.K., 1981, ARA\&A 19, 373

Meier D.L., Jauncey D.L., Preston R.A., et al., 1989, AJ 98, 27

Morganti R., Fosbury R.A.E., Hook R.N., et al., 1992, MNRAS 256 , pp. $1-5$

Pacholczyk A.G., 1970, Radio Astrophysics, Freeman, San Francisco, p. 99

Quinn, 1982, PhD Thesis, Australian National University

Reich P., Reich W., 1988, A\&AS 74, 7

Schreier E.J., Feigelson E., Delvaille J., et al., 1979, ApJ 234, L39

van Albada T.S., Kotanyi C.G., Schwarzschild M., 1982, MNRAS 198, 303 